\title{
Study of the current performance of the European power transformer fleet
}

\author{
F. Ortiz ${ }^{1}$, C. Fernández ${ }^{1}$, C. Olmo ${ }^{1}$, J. Carcedo ${ }^{1}$, C. Renedo ${ }^{1}$, and F. Delgado ${ }^{1}$ \\ ${ }^{1}$ Department of Electrical and Energy Engineering \\ E.T.S.I.I., Cantabria University \\ Avenida Los Castros, 39005 Santander (Spain) \\ Phone/Fax number:+0034 942 201374, e-mail: ortizfa@unican.es
}

\begin{abstract}
In many countries, diverse legislations are today asking for more rational use of energy. For this reason, several initiatives are nowadays promoting a reduction of the overall energy consumption. The European Union has published a Directive 2009/125/EC which fix eco-design requirements to energy-related products. One of these products is power transformers which are a basic piece of generation, transmission, distribution and consumption of electrical energy. The operation of these machines implies the transformation of energy, a part of this energy is dissipated in electrical and magnetic circuits. The amount of losses produce by transformers is defined during the design of these machines. So, they must comply with the above EU Directive. In fact, the Official Journal of the European Union has set a list of energy efficiency limits to be fulfilled by new machines sold in Europe (Commission Regulation 548/2014). This article presents three parts. The calculation of the maximum performance is presented in the first part. This is independent of the load percentage, the impedance and the voltage level. A description of the European fleet of power transformer, up to 100MVA is presented on the second section. In the last part of this work, the efficiency of the fleet is analysed using the maximum performance concept and considering the EU Regulation.
\end{abstract}

\section{Key words}

Power transformer, Performance, Energy Losses, $\mathrm{CO}_{2}$ emissions

\section{Introduction}

Transformers are a very important part of electricity distribution networks. They change this type of energy from a voltage level to another. The number of machines in operation worldwide is very large, it must be noted that electrical energy change the voltage level an average of four times between generation plants and consumers. Diverse types of machines are needed in industrial, transmission and distribution networks, with different values of power and operating voltages.

The energy efficiency or the amount of losses in electrical systems ranges between $3.7 \%$ and $26.7 \%$ of the total electricity consumed, depending on the part of the world [1]. Considering that transformers use to be energized every day throughout the year and taking into account that they operate for an average of 30 years, these devices are the second generator of losses in the electrical grid [1-3]. Three main types of losses are found in a transformer: noload losses, load losses and cooling losses [1, 5-6]. Therefore, when the performance of transformers is improved, many positive consequences can be observed; increased supply security, reduction of natural resources need, lower cost of electricity, etc.

This article studies energy efficiency levels of a power transformers fleet by means of maximum performance of each machine. This value is obtained when the load connected to the transformer correspond to the optimum point. The maximum performance is not influenced by the amount of load, the impedance or the voltage of the machine. Thanks to this characteristic, the maximum performance is useful for the comparison between different units.

In the next sections of this article the following points will be studied: the theoretical calculation of the performance of power transformers, the basic features of a large database of power transformers, the levels of energy efficiency of these machines.

\section{Performance of power transformers}

The losses in these machines have a couple of components; the no-load losses $\left(\mathrm{P}_{0}\right)$ produced in the magnetic circuit, and load losses $\left(\mathrm{P}_{\mathrm{k}}\right)$, produced in the electric conductors.

$$
\begin{aligned}
& \text { No }- \text { Load losses }=P_{0} \\
& \text { Load losses }=P_{k}=C^{2} \times P_{s c}
\end{aligned}
$$

$\mathrm{C}$ corresponds to the loading ratio of the transformer. $\mathrm{P}_{\mathrm{sc}}$ is found in the short circuit test. The performance of these machines $(\eta)$ can be obtained from two data: the output power $\left(\mathrm{P}_{2}\right)$, the input power $\left(\mathrm{P}_{1}\right)$. The total losses $\left(\mathrm{P}_{\mathrm{P}}\right)$ in transformers are determined by the difference between $\mathrm{P}_{1}$ and $\mathrm{P}_{2}$.

$$
\eta=\frac{P_{2}}{P_{1}}=1-\frac{P_{P}}{P_{1}}=1-\frac{P_{P}}{P_{2}+P_{P}}
$$




$$
\eta=1-\frac{P_{0}+C^{2} P_{s c}}{P_{2}+P_{0}+C^{2} P_{s c}}
$$

The impedance, the loading ratio or the voltage level may affect the losses value in similar transformers. This article tries to compare a fleet of transformers in terms of energy efficiency level; so that the maximum performance associated to each transformer design is proposed as value which has no dependence on operation parameters. This maximum performance is reached when load losses are equal to no-load losses. In this point, the loading ratio of the transformer is considered optimum $\left(\mathrm{C}_{\mathrm{opt}}\right)$ :

$$
\begin{aligned}
& P_{0}=C_{o p t}^{2} x P_{s c} \\
& C_{o p t}=\sqrt{\frac{P_{0}}{P_{s c}}}
\end{aligned}
$$

Considering this optimum load, the maximum performance of a unit can be calculated.

$$
\eta_{\max }=1-\frac{P_{0}+C_{o p t}^{2} P_{s c}}{P_{2}+P_{0}+C_{o p t}^{2} P_{s c}}
$$

Taking into account a unity power factor, any group of units can be compared from the energy efficiency point of view.

\section{Case study: energy efficiency of European power transformers}

The power transformers studied ranges from 4 to 100MVA. They are oil-immersed type with voltage greater than $36 \mathrm{kV}$. Fig.1 shows the four types of applications of the fleet. As can be seen, the units studied belong mainly to utilities. This survey contains data of 6853 power transformers, representing approximately $10 \%$ of the European population. All these machines are currently in operation in the following countries: Croatia, France, Italy, Norway, Poland, Portugal, Romania, Spain, Sweden and United Kingdom.

In Fig.2 is showed the energy efficiency or maximum performance of the transformers sampled $\left(\eta_{\max }\right)$. In Fig.3, the performance levels found in this transformers population is compared with the limits set by the EU Commission Regulation 548/2014 of 21 May 2014 on implementing Directive 2009/125/EC of the European Parliament and of the Council with regard to small, medium and large power transformers.

Two sets of maximum performance limits to be overcome by new transformers are presented in the previous EU regulation; the lowest one affects to any unit purchased from the first of July 2015 and the highest one affects to any unit purchased from the first of July 2021. In Fig.3, the limits proposed are drawn, the lowest one, T1, in red color and the highest one, T2, in blue color. Later, instead of using the performance representation shown in Fig.2, trend lines have been drawn considering the average performance sampled for each rated power. This way, the current energy efficiency situation of the European fleet could be observed and compared with the legal limits.

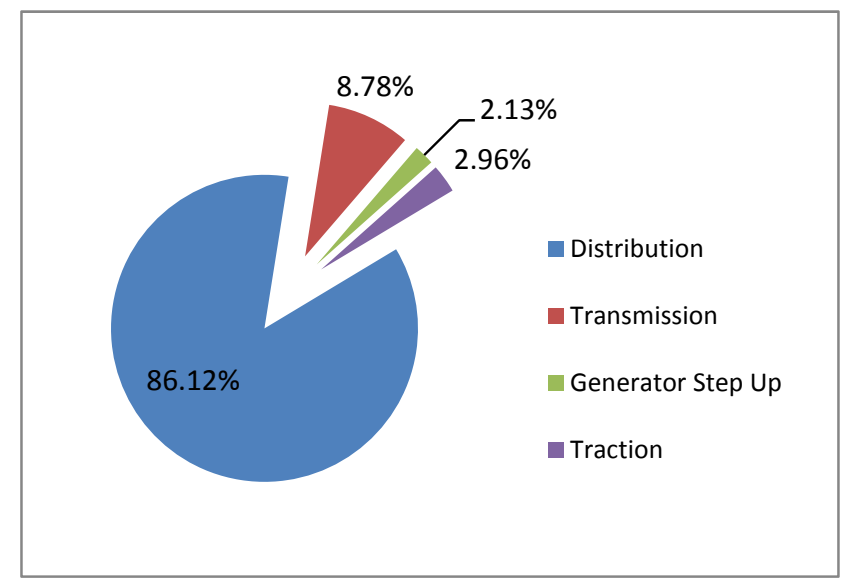

Fig. 1. Percentage of units analysed per category.

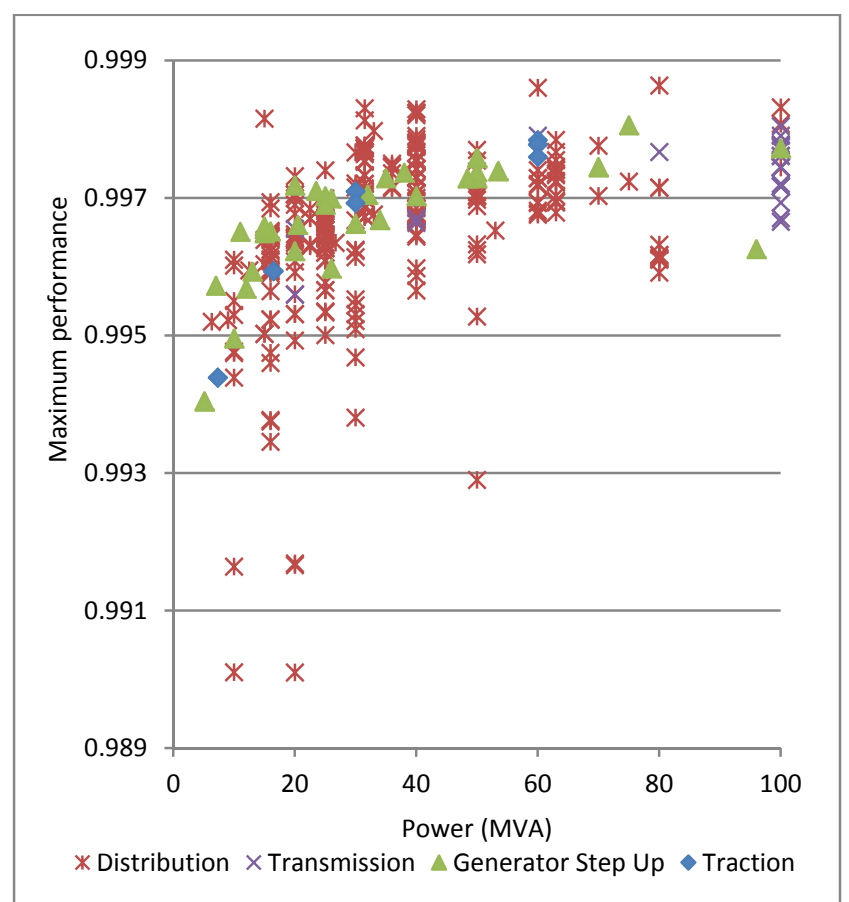

Fig. 2. Maximum performance of the units analysed.

\section{Capitalization of losses of power transformers}

Transformers with reduced level of losses are also more expensive than those less efficient. This is due to the higher costs of materials needed for manufacturing the machine. However, if one takes into account all the energy transformed by a machine yearly and the cost of the losses, the extra cost associated with a high efficient machine is amortized in few years, thanks to the increased performance. In this manner, companies that use capitalization of losses when buying a new transformer do not try to minimise transformer losses, instead it is used to minimise the investment required to obtain the greatest energy savings for the least cost, arising from lower loss transformers. This in turn results in the selection of transformers whose losses are economically optimal, but not minimal. Hence capitalization of losses could be considered as an efficient way to optimize transformer design in accordance with the customer needs. 


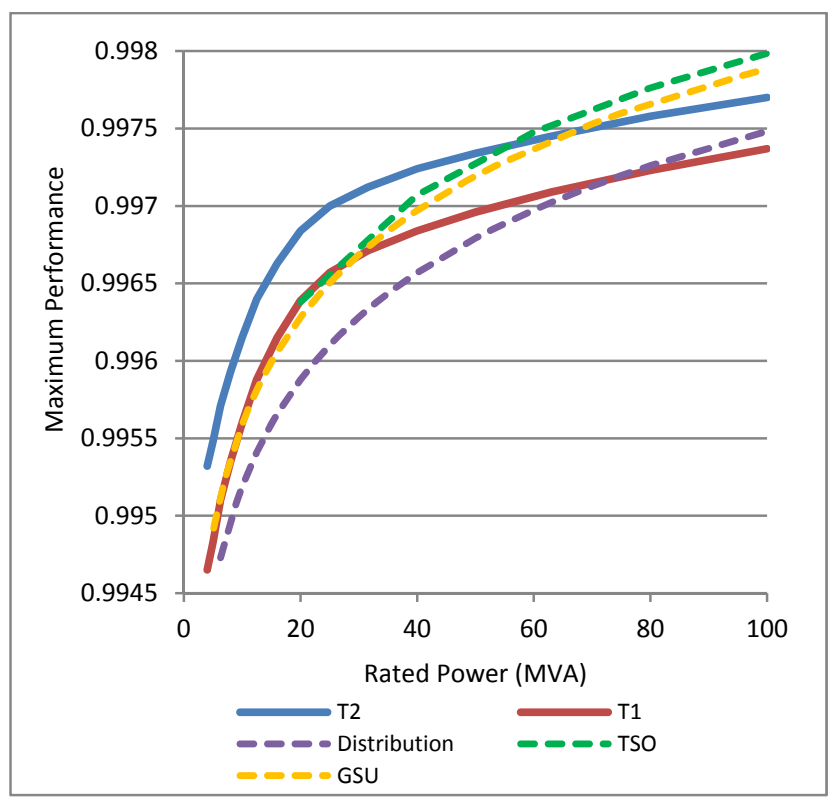

Fig. 3. Average maximum performance of Distribution, TSO and GSU units compare with legal limits.

Nevertheless the definition of the costs to be included in the capitalization of losses needs to be consistent in order to truly optimise benefits to society and to take externalities into account. This is especially true for TSOs or Utilities which are regulated, and where the regulator's goal is to optimise utility investments in the wider context of society.

Accordingly the initial cost of the Transformer should include all associated lifetime costs such as installation, maintenance, end of life disposal etc. In practice the Specification defines the criteria of size and weight within which the transformer is to be designed, the disposal method required and the maintenance regime, so that once the Specification is met the installation and other associated costs for all transformers would be the same and would not need to be included in initial costs.

However if the Specification was left open then the cost and size of the foundation, the design costs to accommodate different heights of transformers, different bunding costs for different oil volumes, Planning Permissions, etc, would all need to be included in Initial Costs.

\section{A. Capitalization formula}

To be fully relevant capitalization shall be based on the forecast cost of energy each year of the transformer life and, on the actual losses at this period, and relate these future cash flows to today using the appropriate discount rate. Unfortunately the parameters required are not easily available and are often too uncertain in value to determine the capitalization values with certainty.

The losses used for capitalization evaluation should include the cooling losses, with the no load losses for the part always on, and with the load losses for the variable part. The Total Ownership Cost being then defined by:

$$
T O C=I C+A \cdot(P O+P C O)+B \cdot(P k+P C S-P C O)(9)
$$

Where:
IC is the cost of the transformer. This cost may include installation costs such as foundation and erection costs (to be better be evaluated because an offer could be very complicate in such case).

$P 0$ is the no load losses measure at rated voltage and rated frequency, on the rated tap summed

$\mathrm{PCO}$ is the cooling power needed for no load operation

$P k$ is the losses due to load measured at rated current and rated frequency on the rated tap at a reference temperature equal to $20+$ Guaranteed average copper temperature rise. If the transformer is equipped with a third winding designed to carry a load in a three winding operation, the load losses shall be based on the three winding operation.

PCS is the total cooling power needed for operation at rated power (including three winding operation if any)

$A$ is the cost of capitalization of no load losses

$B$ is the capitalization cost of the losses due to load

\section{1) Calculation of factor $A$}

The no load losses and their associated cooling losses are present as soon as the transformer is energized therefore the capitalization cost is the valorisation cost of energy multiplied by the operating time over the full life expectancy of the transformer as shown:

$$
A=\sum_{j=1}^{n} \frac{O_{0 j}{ }^{*} C_{j}}{\left(1+i_{j}\right)^{j}}
$$

Where:

$\mathrm{O}_{0 j}$ is the operating time of the transformer at year $\mathrm{j}$ in hours

$C_{j} \quad$ is the valorization of the energy at year $\mathrm{j}$ in $€ / \mathrm{Wh}$ if losses are expressed in $\mathrm{W}$

$i_{j} \quad$ is the discount rate at year $\mathrm{j}$ in per unit

$n \quad$ is the life expectancy of the transformer in years

\section{2) Calculation of factor $B$}

B is the capitalization cost of the losses due to load and it is highly dependent on the load profile. The load of a transformer can usually be split between fix load which is constant and present all year round and affine load which depends on ambient conditions and may be present only part time. For the sake of calculation it is useful to define the average loss load factor $(\mu)$ as the square of the r.m.s. value of the instantaneous load factors by:

$$
\mu=\frac{1}{T} \int_{0}^{T}(k(t))^{2} d t
$$

Where:

$\mathrm{T}$ is equivalent to one year if $\mathrm{k}(\mathrm{t})$ is defined per hours $\mathrm{T}$ is $8760 \mathrm{~h}$; if $\mathrm{k}(\mathrm{t})$ is defined per minutes $\mathrm{T}$ is $525600 \mathrm{~min}$.

$\mathrm{k}(\mathrm{t})$ is the loading factor as a function of time

The load losses capitalization cost comes as the sum of the loads factors multiplied by the cost of energy and corrected by the increase of load and the increase of transformer installed base. In the following equation 12 
below the losses are spitted in two parts each one affected by its time base utilization:

$$
B=\sum_{j=1}^{n} \frac{\mu \cdot C_{j} \cdot\left(O_{a j} \cdot T_{a j}+O_{f j} \cdot T_{f j}\right)}{\left(1+i_{j}\right)^{j}}\left(\frac{1+C_{\mu j}}{1+C_{a j}}\right)^{2 j}
$$

Where:

$\mu \quad$ is the average load loss factor as defined above

$\mathrm{Cj}$ is the valorization of the energy at year $\mathrm{j}$ in $€ / \mathrm{Wh}$ if losses are expressed in $\mathrm{W}$

$\mathrm{i}_{\mathrm{j}} \quad$ is the discount rate at year $\mathrm{j}$ in per unit

$\mathrm{O}_{\mathrm{aj}}$ is the operating time of the transformer at affine load during year $\mathrm{j}$ in hours

$\mathrm{O}_{\mathrm{fj}}$ is the operating time of the transformer at fixed load during year $\mathrm{j}$ in hours usually 8760 hours if the transformer is operated all year round

$\mathrm{T}_{\mathrm{aj}}$ is the share of affine load in the total load loss factor at year $\mathrm{j}$

$\mathrm{T}_{\mathrm{fj}}$ is the share of fixed load in the total load loss factor at year $\mathrm{j}$

$\mathrm{Taj}+\mathrm{Tfj}=1$

$\mathrm{n} \quad$ is the life expectancy of the transformer in years

$\mathrm{C}_{\mu \mathrm{j}}$ is the rate of load loss factor increase at year $\mathrm{j}$

$\mathrm{C}_{\mathrm{aj}}$ is the rate of installed power increase at year $\mathrm{j}$

Usually $\mathrm{C}_{\mu \mathrm{j}}$ and $\mathrm{C}_{\mathrm{aj}}$ are taken equal to zero which corresponds to a situation where the investment is assessed on the basis that the average loading of transformer as invariant. If this is not the case special care shall be taken to avoid overloading of transformer from a certain year, as if $C_{\mu j}$ is greater than $C_{a j}$ the final factor is greater than one.

The previous approach is true if the investment is assessed on the basis that the average loading of transformer as invariant. In other case, a correction depending on the increase of load and the increase of transformer installed base should be considered. [8]

\section{Case study: effect of the capitalization of losses on the energy efficiency of European power transformers}

For this study, 3437 machines belonging to European Transmission System Operators and Utilities were considered. In the following Fig. 4 is shown how many transformers belong to each group and how many of them were acquired with capitalization of losses. $6.49 \%$ of this population of machines have capitalized their losses. Data have been obtained from transformers currently in operation in Norway, Sweden, UK, Spain, Italy, Croatia, Romania. Below, the analysis of the cost assigned to the no-load and load losses by distribution and transmission companies implementing capitalization of losses is evaluated.

Fig. 5 shows how the TSOs allocated higher costs for losses capitalization than Utilities. For example, a 100MVA machine with an investment in losses of $€ 508,800$ provides a performance of 0.99744 , while another 100MVA machine with an investment in losses of $€ 1,094,875$ provides a performance of 0.99776 .
Fig. 6 shows how, for a given power, machines with higher efficiency have lower associated capitalization costs (TOC-IC).

In all considered powers it can be observed the negative slope of the trend of values.

It can be also observed, in the case of 40MVA machines, two groups of costs, one in a lower range M€224-264, and another at a higher range M€367-418.

This is due to different price levels between two countries surveyed.

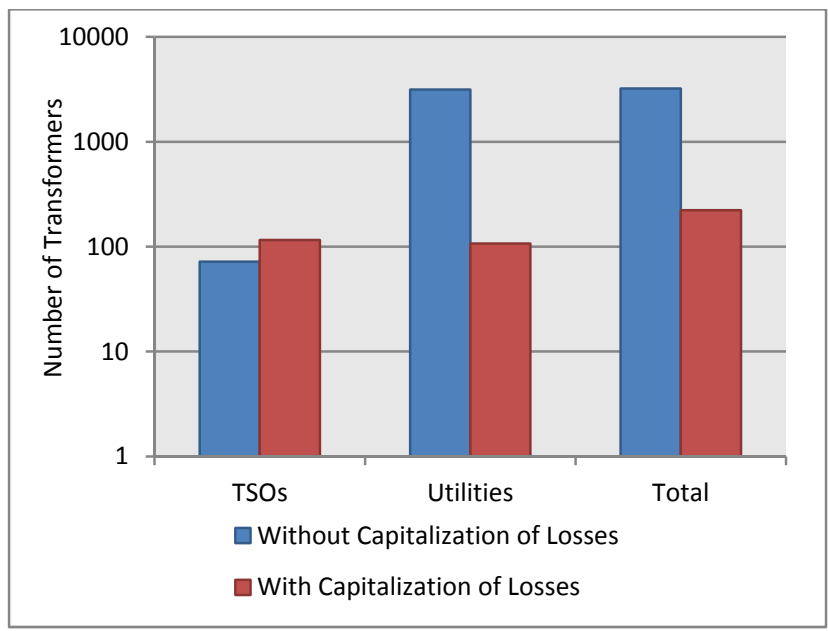

Fig. 4. Classification of the transformers fleet analysed.

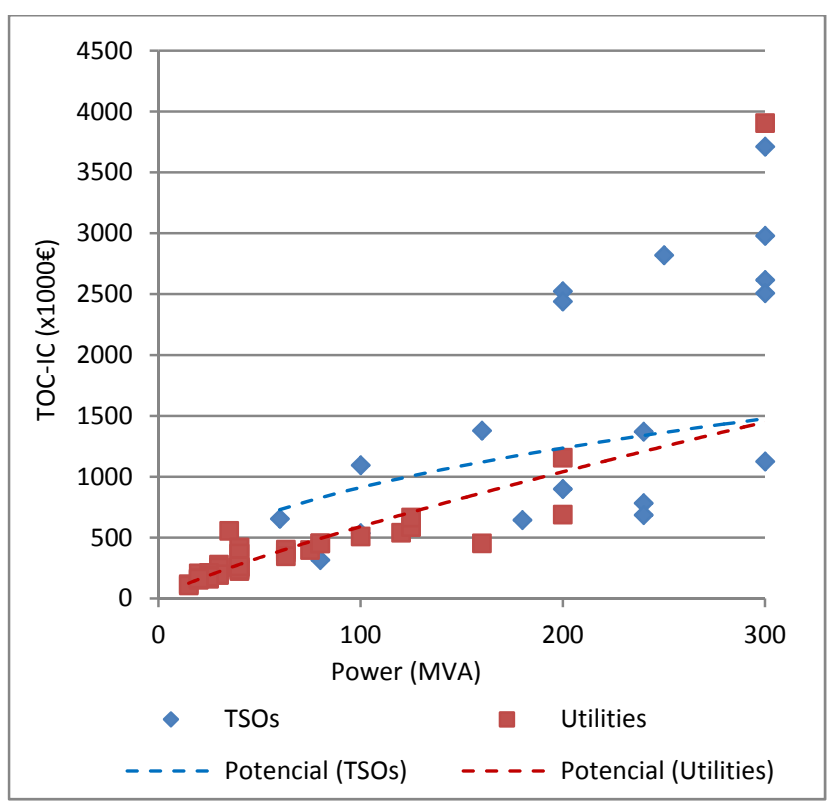

Fig. 5. Investment on losses depending on power size (MVA).

\section{Conclusions}

The final aim of this article was first to observe whether it is necessary to adjust new energy efficiency limits in 2021 (T2) to be achieved for new transformers and to analyse what percentage of the transformers in operation does not achieve these new efficiency targets nowadays. It was observed that transformers with power below 60MVA must be improved for overcoming both $\mathrm{T} 1$ and $\mathrm{T} 2$ limits. Transformers with powers higher 60MVA, TSO and GSU types, present nowadays an average performance better 
than T2 objective. Distribution transformers are those which average performance is lower.

On the other hand, this work has studied the effect of the capitalization of losses during acquisition of new power transformers. Transmission transformers consider higher values when performing capitalization of losses. This results in higher values of energy efficiency. Also, the TOC-IC amount decreases while performance increases, considering a specific transformer power.

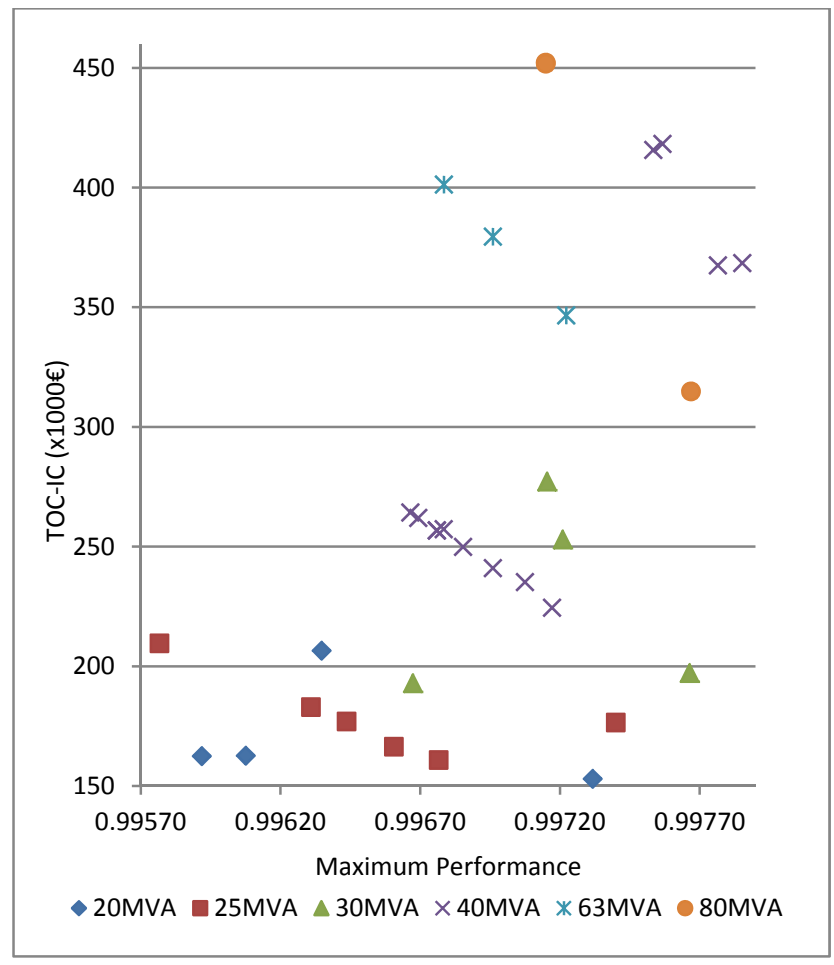

Fig. 6. Investment on losses depending on performance.

\section{References}

[1] Belmans R, Declercq J, De Keulenaer H, Furuya K, Karmarkar M, Martínez M, McDermott M, Pinkievicz I. The Potential for Global Energy Savings from High Energy Efficiency Distribution Transformers. Targosz (ed), Leonardo Energy, European Copper Institute 2005.

[2] Hammons TJ, Kennedy B, Lorand R, Thigpen S, McConnel BW, Rouse S, Prevost TA, Pruess C, Dale SJ, Ramanan VR, Baldwin TL. Future trends in energy-efficient transformers. IEEE Power Engineering Review 1998;18:5-16.

[3] European Copper Institute, The Scope for Energy Saving in the EU Through the Use of Energy-Efficient Electricity Distribution Transformers 1999.

[4] Directive 2009/125/EC of the European Parliament and of the Council of 21 October 2009 establishing a framework for the setting of eco-design requirements for energy-related products.

[5] Georgilakis PS. Environmental cost of distribution transformer losses. Appl Energ 2011;88,3146-55. DOI:10.1016/j.apenergy.2010.12.021

[6] IEEE Loss Evaluation Guide for Power Transformers and Reactors, IEEE Standard C57.120.1991, Aug. 12, 1991.

[7] Frau J, Gutierrez J, Ramis A. Consider the true cost of transformer losses. Transm. Distrib. World 2007;1:50-55.

[8] A. Charalambous, Andreas Milidonis, A.Lazari, A.I.Nikolaidis. Loss Evaluation and Total Ownership Cost of Power Transformers-Part I: A Comprehensive Method. IEEE Transactions on Power Delivery, Vol. 28, No. 3, July 2013. 